\title{
CLINICAL VARIANTS OF DEPRESSION IN ADOLESCENTS IN THE AGE ASPECT
}

\author{
I. Mykhailova, O. Mayorov, N. Goloborodko, T. Matkovska, \\ D. Mitelov, N. Bagatskaya
}

\section{State Institution "Institute of Health Care of Children and Adolescents of the NAMS of Ukraine"}

\begin{abstract}
Background and Aims. Depressive disorders in adolescents can be under the "mask" of pre-puberty and puberty manifestations, somatic and behavioral disorders, creating additional diagnostic difficulties. The interplay between depressive symptoms and puberty crisis manifestations, somatoneurologic history, including endocrine disorders (hypothalamic syndrome, obesity) with puberty period manifestation make depressive disorders diagnostic more complex.
\end{abstract}

Materials and Methods: 256 adolescents aged 12-18 with depressive disorders (DD) were included in the study. Study design included the following aspects: clinical psychopathological, somatic neurological, psychological (children's Depressive Rating Scale Revised - CDRS-R; the Columbia-Suicide Severity Rating Scale (C-SSRS), Toulouse-Pieronne Test to determine the presence and type of a minimal brain dysfunction and Luria's Memorizing 10 words technique, factor analysis of cerebral neurodynamics parameters.
Results: Clinical variants of depression in children at puberty have gender and age differences: in pre-puberty the phobic, somatic variants predominate; in early puberty - behavioral, dysmorphophobia variants predominate; in actual puberty - anxious, apathetic, and behavioral variant predominate. As the age increasing the risk of depression behavioral variant forming both in boys and girls increases; formation of dysmorphic variant in girls in early puberty and phobic and somatic variants ratio decrease. It was found that depression formation in adolescents is realized in the neurotransmitter systems imbalance conditions, which has certain sexual characteristics and determines the variety of depressive disorder clinical manifestations. The role of depression neurophysiological components in children at puberty - neurogenesis emotional-cognitive and cognitive reduced factors, reduction of Kolmogorov-Sinai entropy in frontal leads was determined.

Conclusion: Clinical differentiation of depression progress variants in children in puberty will make it possible to determine the vector of therapeutic intervention and risk prevention of depressive disorders resistant forms developing in young age.

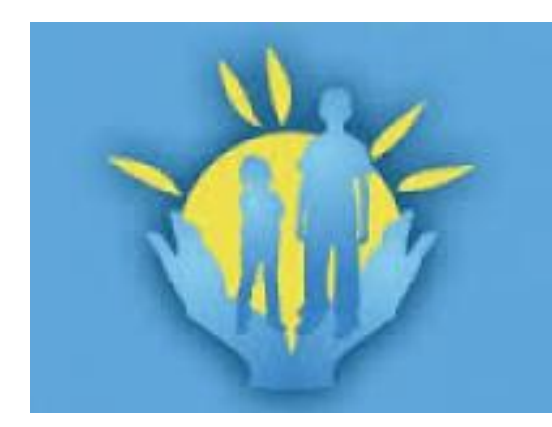

